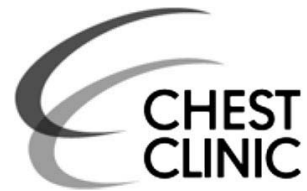

${ }^{1}$ Department of Pulmonary Medicine, Clínica Alemana de Santiago, Universidad del Desarrollo, Santiago, Chile ${ }^{2}$ Department of Radiology, Clínica Alemana de Santiago Universidad del Desarrollo, Santiago, Chile

${ }^{3}$ Department of Pulmonary Medicine, The University of Texas MD Anderson Cancer Center, Houston, Texas, USA

\section{Correspondence to}

Dr Macarena R Vial, Department of Pulmonary Medicine, Clínica Alemana de Santiago, Universidad del Desarrollo, Santiago, Chile;

mrodriguezvi@alemana.cl

Received 19 January 2018 Revised 7 March 2018 Accepted 12 March 2018 Published Online First 23 March 2018

Check for updates

To cite: Vial MR, Horwitz B, Ramos C, et al. Thorax 2018;73:894.

\title{
Massive haemoptysis TB or not TB
}

\author{
Macarena R Vial, ${ }^{1}$ Benjamin Horwitz, ${ }^{2}$ Cristobal Ramos, ${ }^{2}$ Karen Czischke, \\ Georgie Eapen, ${ }^{3}$ Horiana B Grosu ${ }^{3}$
}

A young lifelong non-smoking patient presented to the emergency department after an episode of haemoptysis following a 2-month history of weight loss, cough and fever. He had a history of pulmonary tuberculosis during childhood, for which he completed 6 months of treatment. An initial non-contrast CT of the thorax revealed a right lower lobe opacity suggestive of necrotising pneumonia with an endobronchial lesion in the bronchus intermedius and associated mediastinal and hilar lymphadenopathy (figure 1). A subsequent CT angiogram revealed bilateral hypertrophic bronchial arteries and a $6 \mathrm{~mm}$ intrapulmonary pseudoaneurysm arising from the right bronchial artery (BA), forming the previously described endobronchial lesion. During his initial assessment, massive haemoptysis occurred, prompting emergent transfer to the Interventional Radiology Unit, where an urgent bronchoscopy was carried out. During bronchoscopy, several clots were observed in the right main stem bronchus, bronchus intermedius and a bleeding endobronchial lesion was seen in the bronchus intermedius (figure 2A) for which a selective bronchial balloon blocker was placed. A bronchial arteriography was then performed and showed enlargement and tortuosity of the vessels and marked hypervascularity with the presence of a bronchial pseudoaneurysm (figure 2B). Subsequent embolisation was performed using polyvinyl alcohol particles $300-500 \mu \mathrm{m}$ in size (Cook, Bloomington, USA). After the bleeding was controlled and selective arteriography showed no residual flow, a bronchoalveolar lavage and endobronchial ultrasound with transbronchial needle aspiration was performed at the subcarinal lymph node. PCR was positive for mycobacteria but cultures were negative. Histopathology revealed inflammatory necrosis. In view of the disease severity and despite the fact that PCR may have indicated past disease, the patient was started on antituberculous
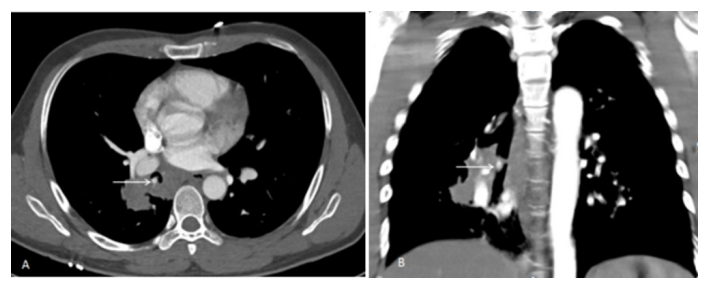

Figure 1 (A) CT scan of the chest axial and (B) sagittal view showing a contrast-enhanced endobronchial lesion in the bronchus intermedius along with associated mediastinal lymphadenopathy and right lower lobe masslike consolidation.
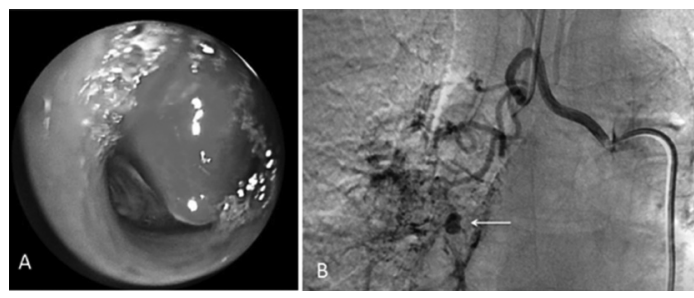

Figure 2 (A) Bronchoscopy image showing an endobronchial polypoid lesion in the bronchus intermedius. (B) Combined right bronchial-intercostal trunk angiogram. Note enlargement and tortuosity of the vessels and marked hypervascularity with the presence of a bronchial pseudoaneurysm (arrow).

treatment with clinical and radiographic improvement within 2 months.

Although bronchial arterial hypertrophy is a common cause of haemoptysis, ${ }^{1}$ BA pseudoaneurysms are uncommon entities ${ }^{23}$ and it is extremely rare to visualise a BA pseudoaneurysm eroding into the airway. BA embolisation is a safe and effective non-surgical treatment for these patients and has become first-line therapy. ${ }^{4}$ Interventional pulmonology techniques such as selective balloon blockers can provide temporary stabilisation permitting definitive treatment. This case highlights the importance of multidisciplinary management in these complex patients to achieve successful outcomes.

Contributors MRV, BH, CR and KC: conception, data acquisition, drafting the manuscript and final approval. HBG and GE: critically revising and final approval.

Funding The authors have not declared a specific grant for this research from any funding agency in the public, commercial or not-for-profit sectors.

Competing interests None declared.

Patient consent Obtained.

Provenance and peer review Not commissioned; externally peer reviewed.

(C) Article author(s) (or their employer(s) unless otherwise stated in the text of the article) 2018. All rights reserved. No commercial use is permitted unless otherwise expressly granted.

\section{REFERENCES}

1 Yoon W, Kim JK, Kim YH, et al. Bronchial and nonbronchial systemic artery embolization for life-threatening hemoptysis: a comprehensive review. Radiographics 2002;22:1395-409.

2 Restrepo CS, Carswell AP. Aneurysms and pseudoaneurysms of the pulmonary vasculature. Semin Ultrasound CT MR 2012;33:552-66

3 Sopko D, Smith T. Bronchial artery embolization for hemoptysis. Semin Intervent Radiol 2011;28:048-62.

4 Ramakantan R, Bandekar VG, Gandhi MS, et al. Massive hemoptysis due to pulmonary tuberculosis: control with bronchial artery embolization. Radiology 1996;200:691-4. 